\title{
Marcadores para la Realidad Aumentada para fines educativos
}

\author{
María Reina Zarate Nava \\ Universidad Tecnológica del Centro de Veracruz \\ ma.reina.zarate@gmail.com
}

Cecilio Francisco Mendoza González Universidad Tecnológica del Centro de Veracruz cecilio.francisco.mendoza@gmail.com

Honorato Aguilar Galicia

Universidad Tecnológica del Centro de Veracruz aguilargh@hotmail.com

Juan Manuel Padilla Flores

Universidad Tecnológica del Centro de Veracruz jmpf33@hotmail.com

Resumen: La Realidad Aumentada (AR) es considerada por algunos expertos como la tecnología en crecimiento para el 2014, en diferentes sectores: ingeniería, educación, juegos y negocios, siendo este último el más usado en él 2013. Sin embargo en el sector educativo la AR se presenta en algunos proyectos que se han desarrollado para diferentes áreas de las ciencias, como Química, Física y Matemáticas, lo anterior con el objetivo de facilitar el proceso de Enseñanza-Aprendizaje. En el presente artículo se presenta el modelo de patrones utilizado para un Proyecto AR enfocado en Química, el cual presenta un conjunto de patrones que fueron valorados para facilitar el 
rastreo de los objetos y a su vez el entendimiento de algunos materiales de los laboratorios de Química I.

Palabras clave: realidad aumentada, patrones, marcadores, laboratorios virtuales, Química virtual, proceso de aprendizaje.

\title{
Augmented Reality Markers for educational purpose
}

\begin{abstract}
Augmented Reality (AR) is considered by some experts as a growing technology in 2014, in sectors like engineering, education, gaming and business, but business AR has been the most used in 2013. However, in the education sector, AR appear in some projects that have been developed for areas such as science, chemistry, physics and math, all of them with one purpose, to facilitate the learning process. This paper shows a model of patterns used for an AR project focused on chemistry, which presents a set of patterns that were assessed to facilitate tracking of objects and understanding some material of Chemistry lab I subject.
\end{abstract}

Keywords: augmented reality, patterns, markers, virtual labs, Chemistry virtual, learning process.

\section{Introducción}

El poder computacional que se tiene actualmente ha permitido desarrollar nuevo software con tecnología de realidad aumentada para apoyo didáctico, el cual busca asegurar que la impartición de los planes de estudio sea completa, así mismo hacer que la trasferencia de los conceptos sea de manera clara y asertiva, permitiendo al estudiante desarrollar un conocimiento preciso del concepto del fenómeno observado.

Desde hace una década la programación de Software de realidad aumentada ha tomado gran interés en diferentes sectores tales como: educativo, industrial, servicios y recreativos etc. Los primeros indicios de la realidad aumentada (AR, por sus siglas en inglés) se encuentran en el mundo cinematográfico y en la fotografía (Abud Figueroa, 2012). Se define a la realidad aumentada como la integración de objetos virtuales y se menciona que su nacimiento fue en los 
años setenta, con inventos como el "Sensorama" y el "head-mounted Display", los cuales dieron entrada a las primeras publicaciones sobre el tema y la inversión de las compañías en investigación e impulsar el surgimiento de los ahora Smartphone, los cuales actualmente son el soporte más popular para la AR (Arroyo-Vázquez, 2011). Es importante mencionar que la AR no está enfocada a los Smartphone, sino a cualquier dispositivo que tenga integrada una cámara y una pantalla para mostrar el resultado y un software para detectar las referencias y superponer los objetos.

En el campo de la educación, se tienen actualmente distintas aplicaciones de realidad aumentada como: libros aumentados como el "MagicBook" o "El Libro de los Hechizos" ( Sony Computer Entertainment Europe, 2013), y en México se ha aplicado en diferentes sectores del gobierno como el del fomento a la lectura con los "LibroPuertos" en la Ciudad de México, D. F. pero nulos en el caso de Química para secundaria. Es importante señalar que actualmente en México no se cuenta con aplicaciones enfocadas al área de Química, pero a nivel internacional existe una empresa que ha trabajado con proyectos aumentados orientados a las ciencias, el proyecto se denomina LearnAR ( http://www.learnar.org/chemistry.html) y su funcionamiento consiste en mover un marcador que representa un metal y un marcador de solución para que se visualice si existe una reacción en el metal.

En el presente artículo se muestra el desarrollo de marcadores, los cuales son imágenes que una aplicación AR puede reconocer fácilmente (Craig, 2013) y permiten incorporar al entorno real una serie de objetos a través de la cámara; de esta manera se busca generar un laboratorio virtual para realizar prácticas de Química I con el objetivo de mejorar el proceso enseñanza-aprendizaje e incrementar el nivel académico de los estudiantes de nivel Secundaria, lo cual se verá reflejado en la prueba internacional del Programa Internacional de Evaluación de Estudiantes (PISA, por sus siglas en inglés). 


\section{Trabajos relacionados}

Se han realizado distintos proyectos en el campo de la educación, los cuales buscan la adopción del AR en entornos académicos. Dichas aplicaciones se desarrollan para mejorar la educación y la eficiencia de la formación de los estudiantes (Lee, 2012). Uno de los proyectos orientados al área de la Química es el propuesto por Fjeld y Voegtli denominado "Augmented Chemistry"; esté consiste en un entorno interactivo de trabajo para moléculas en $3 \mathrm{D}$, en donde se muestra a los estudiantes en que consiste un átomo o una molécula a través de la AR (Fjeld, 2002). Los marcadores del anterior proyecto consisten de un patrón sencillo en color blanco y negro, donde no se muestra ninguna relación con la Química, la sencillez del patrón se debe a que en total solo se trabajaron con 6 marcadores que colocaron en un cubo.

Otro de los proyectos es el de la empresa "The Schools Network" de Inglaterra, que contiene un conjunto de aplicaciones en el proyecto "LearnAR" (Specialist Schools and Academies Trust, 2013), en el cual uno de los proyectos que se presenta en el área de Química es el de "Reactividad", este muestra una serie de metales y su reacción al ser mezclados con una solución. Los marcadores de dicho proyecto contienen un patrón sencillo, el cual consiste de un borde de color y en el centro un cuadro relleno del mismo color. Cada color está relacionado con un metal o solución según corresponda; la poca elaboración en el patrón es debido a que solo trabajan con seis metales y cuatro soluciones. Lo anterior repercute al momento de que un estudiante ve el marcador, esté no le induce nada relacionado con la Química, que es lo que se busca en el modelo propuesto. 


\section{Modelo propuesto}

Como se mencionó en la introducción, el objetivo del proyecto es permitir a los estudiantes realizar la experimentación en diferentes prácticas de Química I mediante un simulador virtual implementando la AR, en el cual puedan llevar a cabo las prácticas sin necesidad de contar con todo el equipamiento, materiales e instrumental que debe contar un laboratorio de Química completamente equipado.

Para cumplir con lo mencionado anteriormente, el primer paso fue determinar los marcadores "markers", los cuales son imágenes únicas que servirán como referencia para cada uno de los elementos del laboratorio. Para determinar el diseño del marcador se analizaron las cantidades que se necesitarían de los mismos, para ello se consideraron los ciento dieciocho elementos de la tabla periódica, así como ochenta y seis instrumentos de laboratorio de Química y además material extra que se puede requerir en las diferentes prácticas, por ejemplo, globos, pastillas efervescentes u otros, obteniendo un aproximado de 210 marcadores diferentes.

Los marcadores necesitan un patrón único, el cual le permitirá a la cámara reconocer y determinar el objeto u objetos que debe mostrar. El primer patrón generado con un nivel de una estrella (eficiencia baja) se muestra en la Figura 1 , el cual fue descartado porque no permite generar de manera aleatoria cierto número de marcadores, dado que tendía a repetir patrones. Este marcador sólo contiene un patrón sencillo, formado por una cuadricula y utilizando una escala de grises, también se le incorporó la figura del material con ningún patrón en su interior y en la parte inferior se colocó el nombre del equipo sobre un fondo blanco. 


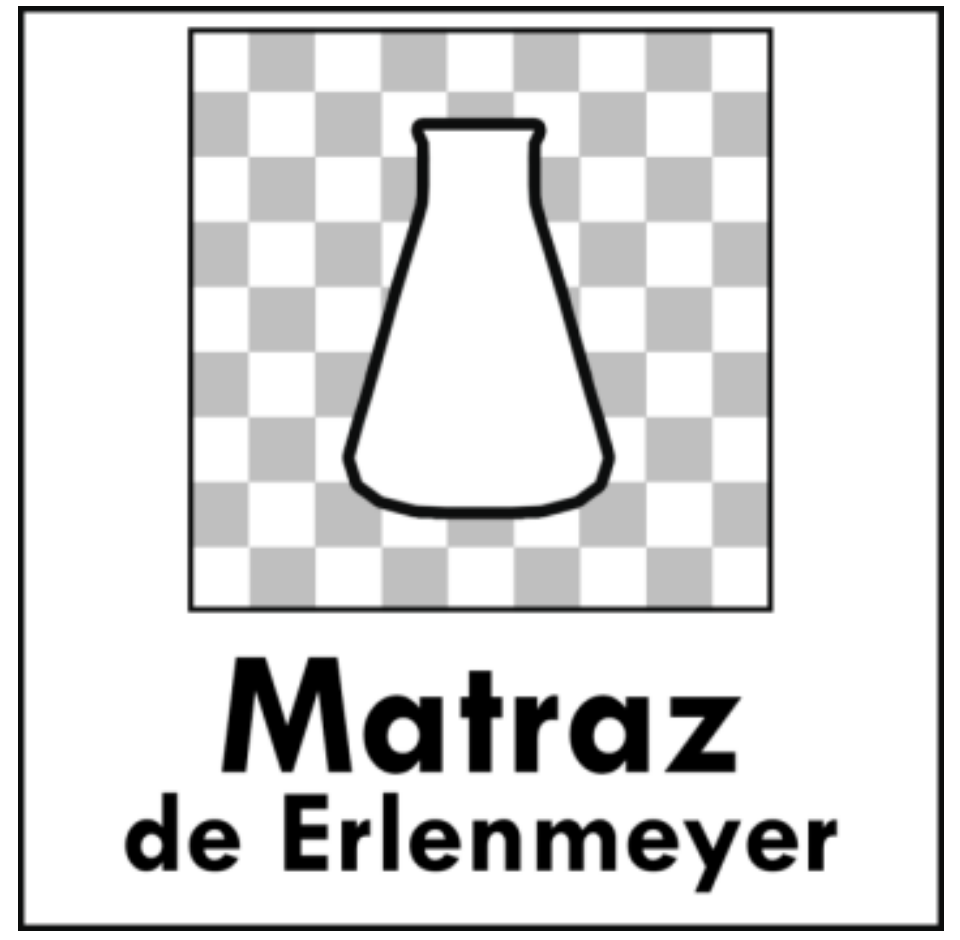

Figura 1.Primer marcador generado para el proyecto de Laboratorio Virtual.

El siguiente patrón mostrado en la Figura 2 es ligeramente diferente en comparación a la Figura 1, este incluye un fondo que cuenta con un patrón propio y se mantuvo la forma del matraz y el nombre completo del mismo. Este patrón fue considerado con un nivel medio de eficiencia, dado que obtuvo 5 estrellas según la evaluación realizada con el software de evaluación "Target Manager" de Vuforia. 


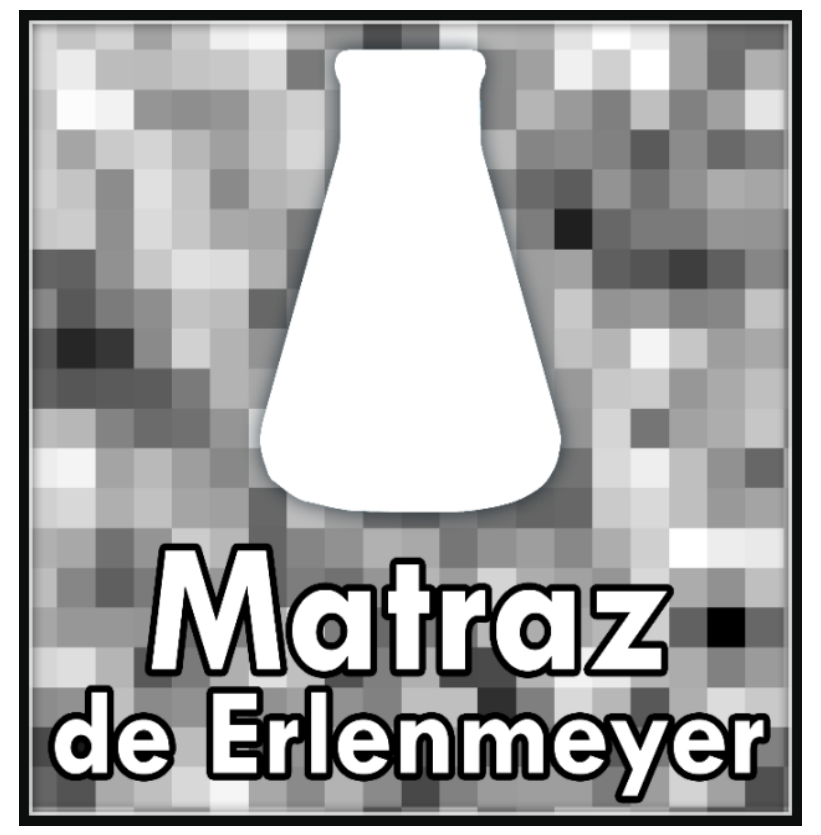

Figura 2.Segundo marcador generado para el proyecto de Laboratorio Virtual.

En la Figura 3, se muestra el patrón que actualmente se utiliza en la aplicación, este patrón incluye el logotipo del proyecto, el equipo que se va a utilizar cuyo fondo ya cuenta con un patrón propio, el nombre del equipo y el fondo base se encuentra también con un patrón en escala de grises. Cabe señalar que los colores no influyen en la eficiencia del marcador en la AR. 


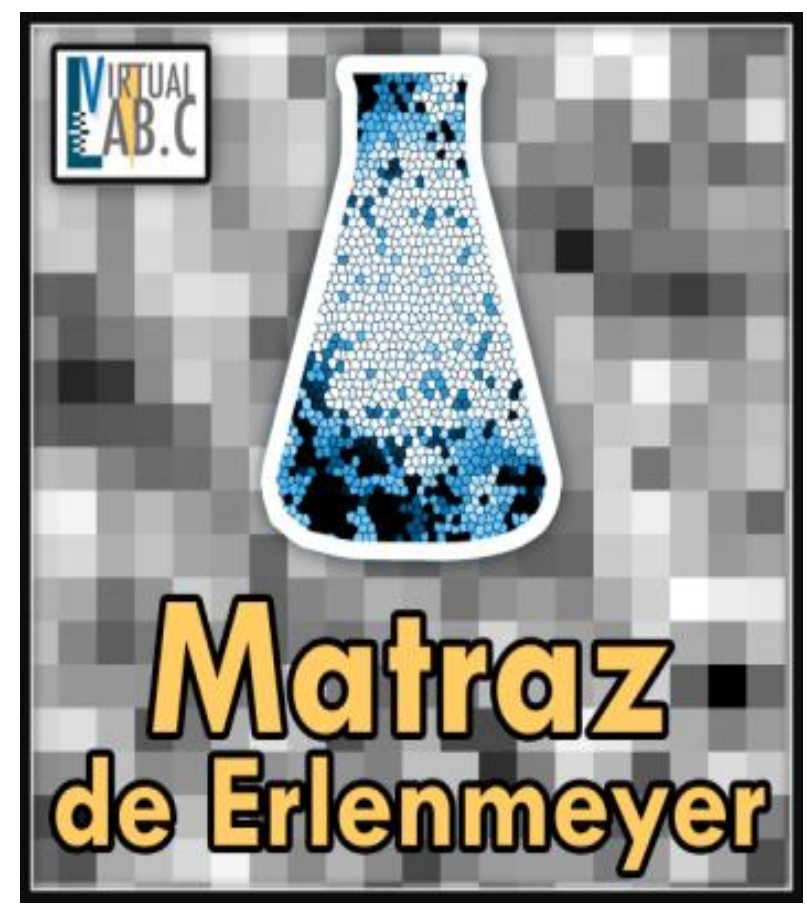

Figura 3.Marcador que actualmente se utiliza en el proyecto de laboratorio virtual y cuenta con una evaluación de 5 estrellas.

Actualmente se están trabajando los diferentes equipos del laboratorio, pero con el equipo más delgado, se encontró el problema de no alcanzar el nivel deseado dado que el patrón dentro del equipo no logra apreciar, por ejemplo la pipeta. Por tal razón, se está trabajando en una nueva versión de marcador, donde se le incorpora un nuevo patrón para permitir la generación de $n$ marcadores. Como se aprecia en la Figura 4, el nuevo patrón de fondo es más reducido y se incorpora un patrón diferente de lado derecho de la imagen, esto permitirá diferenciarlos sin importar el grosor del equipamiento o el tipo de material. 


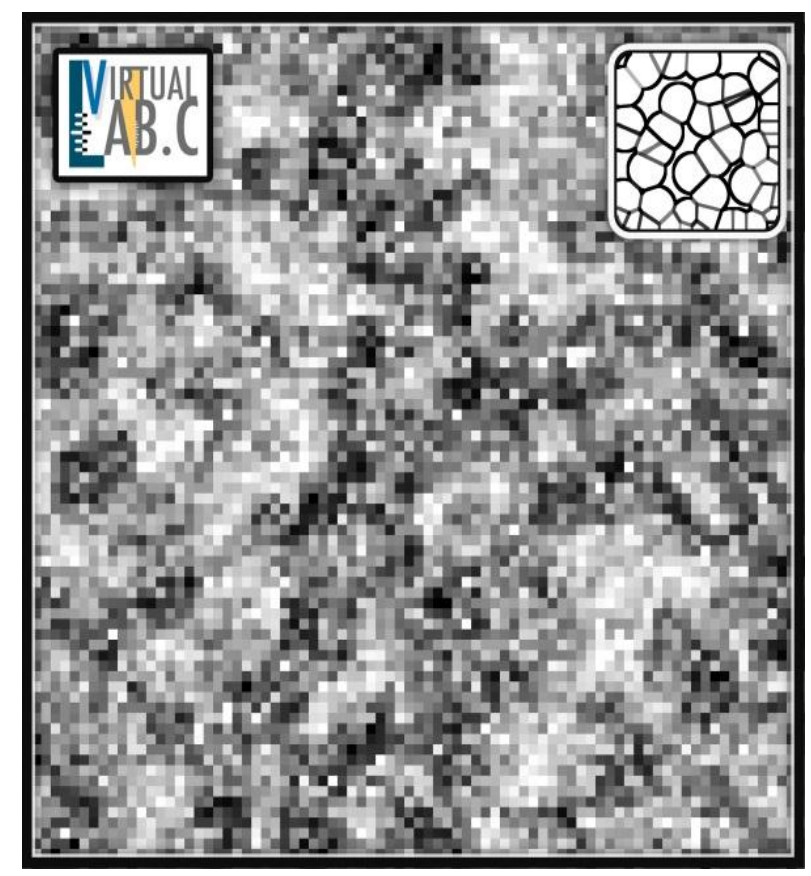

Figura 4.Nuevo marcador, generado por las limitantes que surgen cuando el equipamiento es muy delgado.

Este modelo de patrones en conjunto con el laboratorio virtual, permitirá trabajar con los tres canales de aprendizaje: auditivo, kinestésico y visual. Los alumnos visuales son atraídos por el objeto 3D y el patrón mostrado en los marcadores; los kinestésicos podrán interactuar con cada uno de los objetos y realizar experimentos sin riesgo; los auditivos podrán escuchar un sonido dependiendo de la práctica que esté realizándose. 


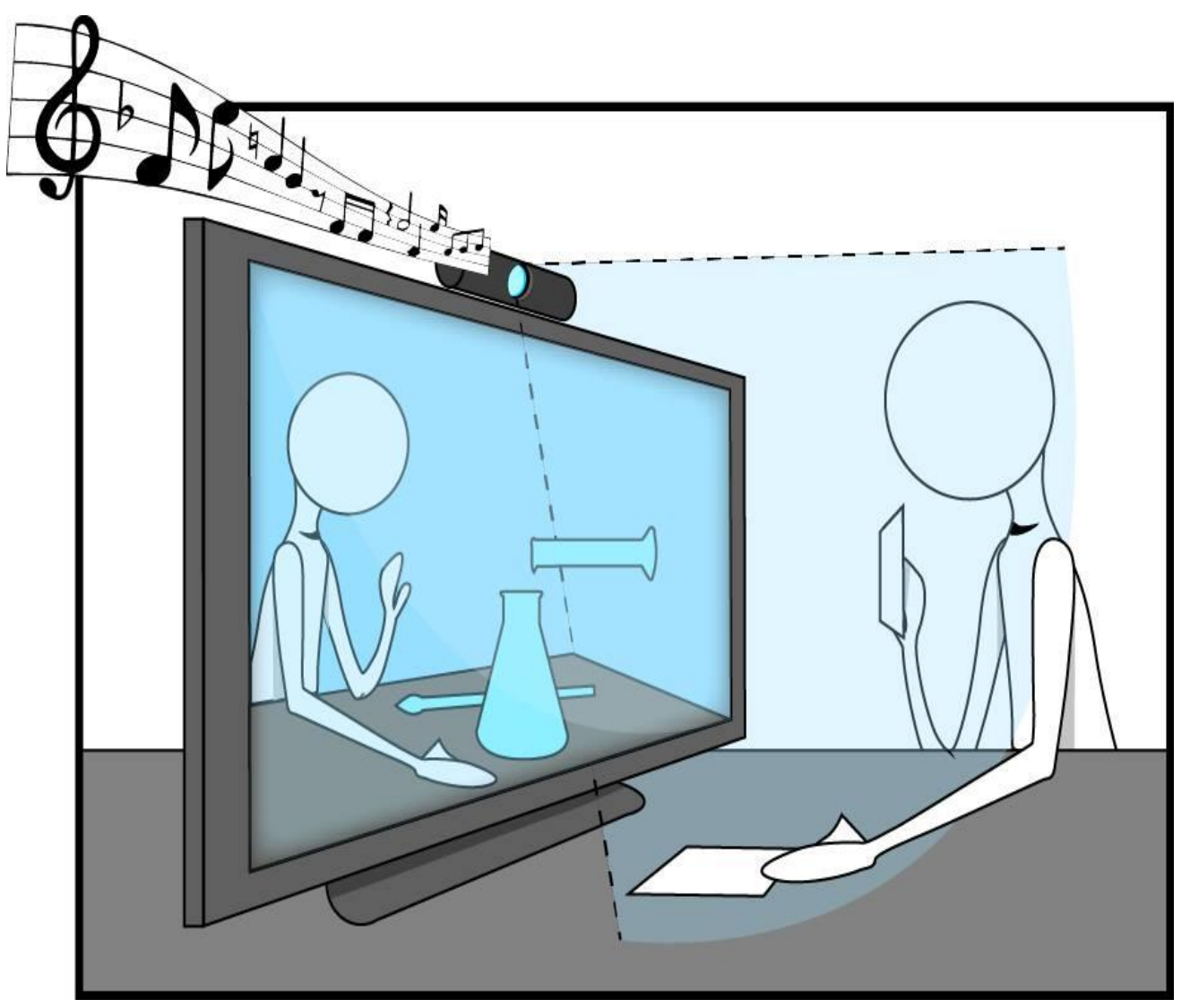

Figura 5.El laboratorio virtual y los canales de aprendizaje.

\section{Resultados}

Del modelo propuesto de marcadores del laboratorio virtual, ya son interpretados de acuerdo con los patrones establecidos mostrándose el material que corresponde. El tiempo de respuesta para la muestra del objeto tridimensional al momento de enfocar el marcador, varía dependiendo de la ubicación de la cámara, obteniendo tiempos de respuesta de hasta 1 segundo; el anterior resultado se obtuvo instalando la aplicación en un Smartphone con sistema operativo Android 4.1.2. El tamaño preferente del marcador para la interpretación del objeto desde la cámara del Smartphone, es de $17 \mathrm{cms} x$ $17 \mathrm{cms}$, aunque un tamaño mínimo para ser detectado por la cámara es de $6 \mathrm{cms} \times 6 \mathrm{cms}$. 
En la Figura 6 se muestran los prototipos realizados, los cuales fueron retroalimentados por especialistas del área, quienes expresan la utilidad de los marcadores incorporando la imagen del material a utilizar, dado que los alumnos lo relacionan con el objeto en la pantalla. El nombre también resulta importante, los especialistas expresan que los alumnos no ubican al material por el nombre, sino por la imagen, trabajando de esta manera con los tres canales de comunicación: kinésico, auditivo y visual.

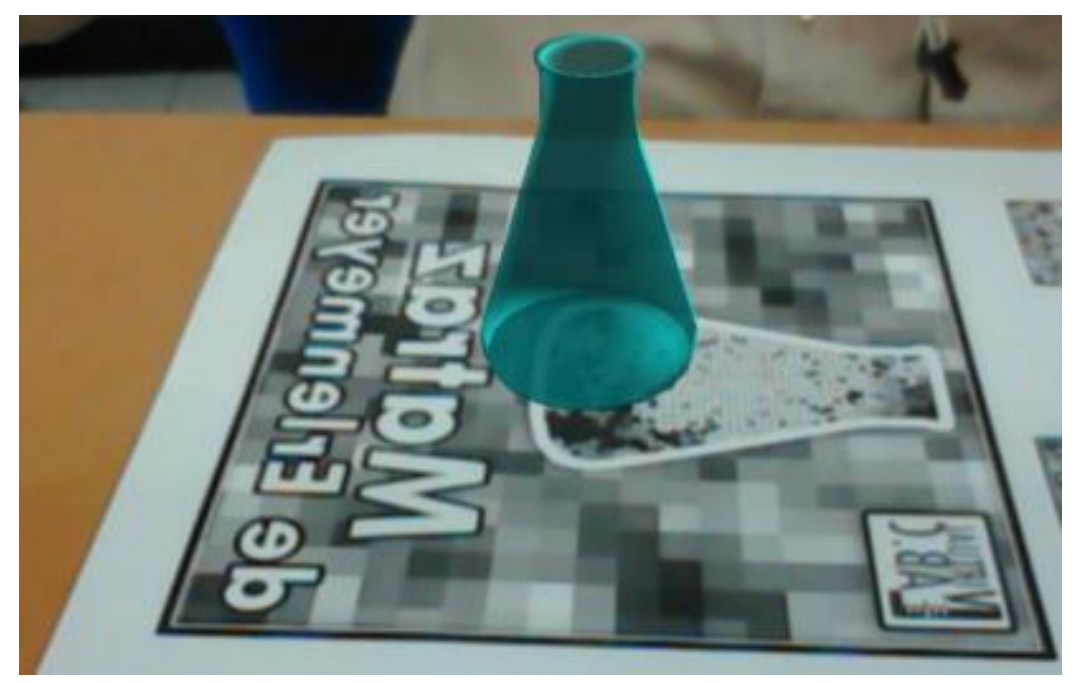

Figura 6.Prototipo inicial de la detección del matraz con base en el marcador.

En la Figura 7, se muestra el marcador de la pipeta y el objeto aumentado, dicho objeto resulto complicado de trabajar debido a su grosor, el cual no permitía enfocar el patrón ubicando dentro de la pipeta. Cómo se mencionó anteriormente, dicho problema fue resuelto con la incorporación de nuevos elementos en el marcador. 


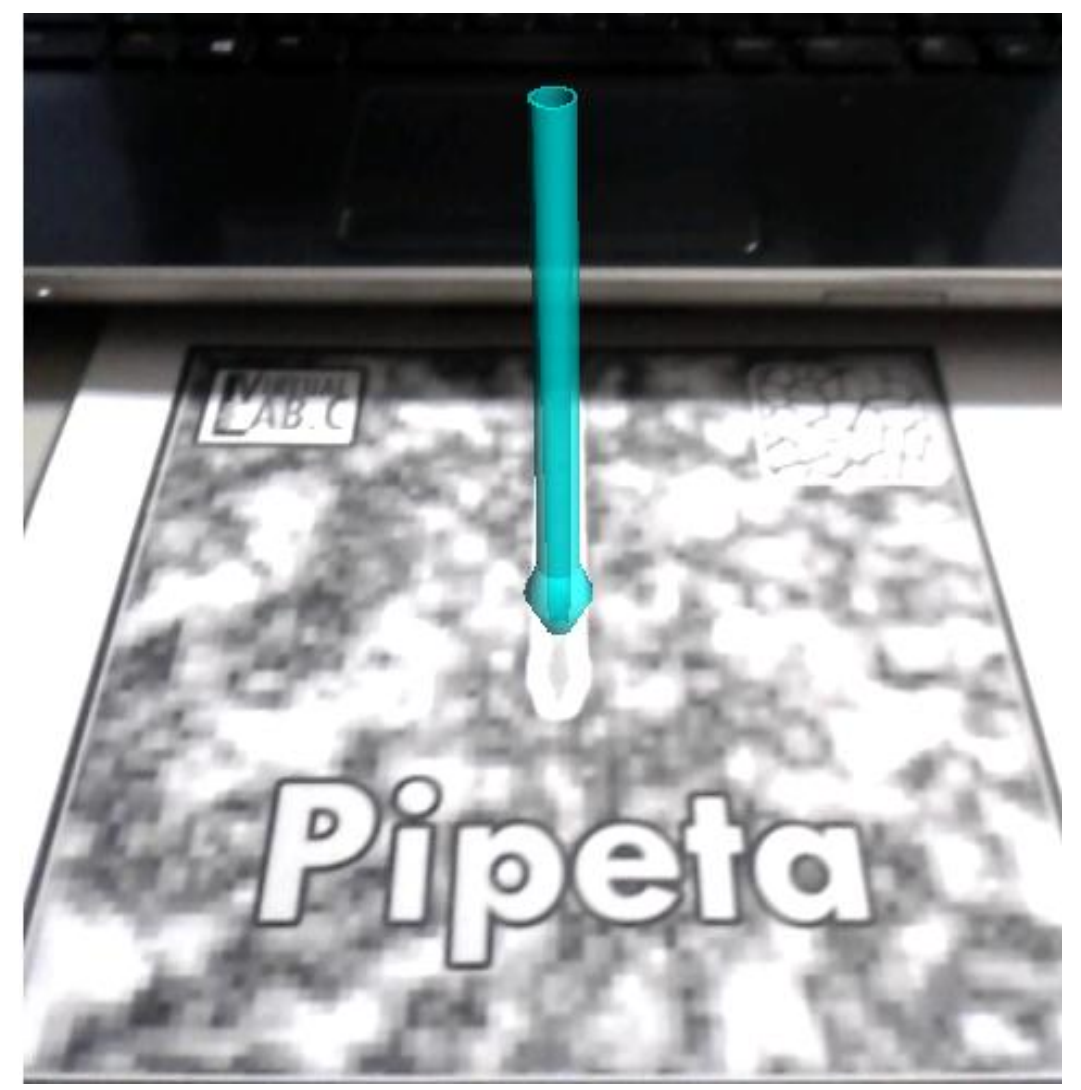

Figura 7.Marcador de la pipeta con patrones más eficientes.

Los objetos anteriores serán mostrados dentro de un laboratorio virtual, dicho escenario permitirá que los alumnos se sientan familiarizados con el ambiente en un laboratorio. Se busca que los materiales como el matraz y la pipeta, se muestren sobre el laboratorio virtual, creando un ambiente aumentado relacionado con la Química.

Los prototipos anteriores, han permitido comprobar la importancia de un patrón eficiente para generación de $\mathrm{n}$ marcadores, permitiendo cubrir un total de doscientos diez, requerido por el proyecto. La elaboración de cada uno de los patrones dio como resultado un marcador que es eficiente, completo, y provee buen nivel de rastreo para realizar la diferencia entre objetos, en comparación con otros marcadores, como se muestra en la Tabla 1. 


\begin{tabular}{|l|c|}
$\begin{array}{l}\text { Marcadores del laboratorio virtual de } \\
\text { Química. }\end{array}$ & $\begin{array}{c}\text { Marcadores Learn AR en su } \\
\text { Proyecto: Chemistry Reactivity. } \\
\text { (Specialist Schools and Academies } \\
\text { Trust, 2013) }\end{array}$ \\
\hline El marcador ofrece buen nivel de rastreo \\
y el patrón permite generar una gran \\
variedad de marcadores. Al estudiante \\
permite relacionarlo con el material del \\
laboratorio de Química.
\end{tabular}

Tabla 1. Tabla comparativa del marcador propuesto con los marcadores del proyecto Learn AR.

Cuando el estudiante realice una combinación de marcadores obtendrá un resultado como el mostrado en la Figura 8 , el cual dependerá de la calidad seleccionada por el usuario. 


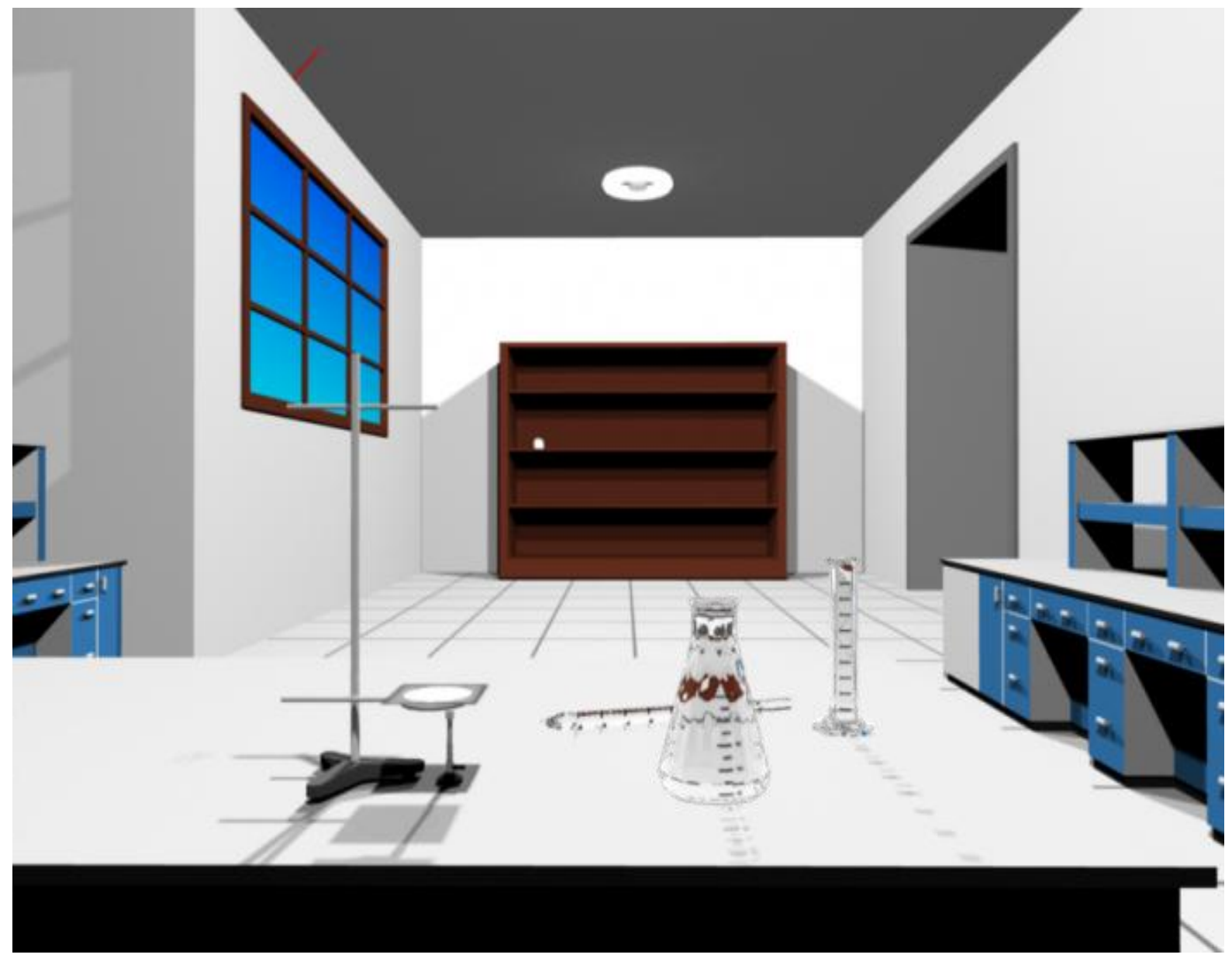

Figura 8. Ambiente aumentado para el laboratorio virtual de Química.

\section{Conclusión}

El modelo presentado de patrones del laboratorio virtual para el desarrollo de han permitido comprobar la importancia de un patrón eficiente para generación de $\mathrm{n}$ cantidad de marcadores, permitiendo cubrir la cantidad requerida por el proyecto. También se ilustraron los patrones y su uso para la representación de los objetos, donde la calidad de los objetos puede ser editada dependiendo de la capacidad del dispositivo en donde se muestren los objetos. 
El uso de estos patrones provee un rastreo de objetos específico, minimizando el error al confundir marcadores en la aplicación, y es una alternativa para el desarrollo de aplicaciones AR que impliquen la generación de $\mathrm{n}$ marcadores. Como trabajo futuro se contempla implementar "Kinect" para la manipulación de los objetos, para que los estudiantes puedan interactuar con los mismos y hacer el ambiente aumentado más real. Además de que el sonido proporcionado por la aplicación pueda variar dependiendo de las acciones realizadas por el estudiante a través del kinect.

\section{Referencias}

Abud Figueroa, M. A. (2012). Modelo de Objetos de Aprendizaje con Realidad Aumentada. Revista Internacional de la Educación en Ingeniería, 1-7.

Arroyo-Vázquez, N. (2011). La realidad aumentada al alcance de todos: creando capas de datos geolocalizados. Anuario ThinkEPI, págs. 269-271.

Craig, A. B. (2013). Understanding Augmented Reality Concepts and Applications. USA: Elsevier.

Bernat, A., \& Catalá, A. (2008). VideoJuegos y Aprendizaje (1 ed.). España: GRAÓ.

Fjeld, M. \&. (2002). Augmented chemistry: an interactive educational workbench. Proceedings of the international symposium on mixed and augmented reality (ISMAR '02).

Lee, K. (2012). Augmented Reality in Education and Training. TechTrends, 1316.

Sony Computer Entertainment Europe. (2013). Wonderbook: EL libro de los Hechizos. Recuperado el 14 de Marzo de 2013, de Sony Computer Entertainment Europe: http://es.playstation.com/bookofspells/

Specialist Schools and Academies Trust. (2013). http://www.ssatuk.co.uk/about/. Recuperado el 10 de 02 de 2013, de http://www.ssatuk.co.uk/

http://www.learnar.org/assets/pdf/LAR_resource_combined.pdf 


\section{Notas biográficas}

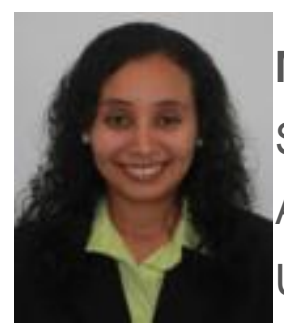

María Reina Zarate Nava estudio la Maestría en Redes y Sistemas Integrados del Laboratorio Nacional de Informática Aplicada y actualmente labora como Profesor-Investigador en la Universidad Tecnológica del Centro de Veracruz. Es programador certificado por parte de Oracle y Microsoft en tecnologías como: ASP.Net, Silverlight y Java; además es mentor certificado por parte de la Universidad Carnegie Mellon. Su interés se encuentra actualmente en la Realidad Aumentada asesorando distintos proyectos que utilicen está tecnología.

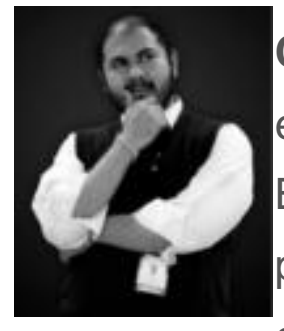

Cecilio Francisco Mendoza González recibió el grado de Master en Animación por parte de la Universidad Pompeu Fabra de Barcelona, los principales intereses radican en la animación de personajes antropomórficos y el desarrollo de historias basadas en el realismo mágico.

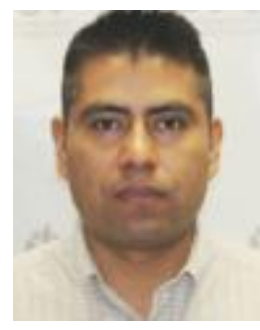

Honorato Aguilar Galicia obtuvo el grado de Maestro en Ciencias de la Computación por parte del Instituto Politécnico Nacional en el Centro de Investigación en Computación, con la realización de una tesis titulada "Extracción automática de información semántica basada en estructuras sintácticas". Ha publicado artículos para el Instituto Politécnico Nacional y su interés actual radica en la investigación de tecnologías de Realidad Aumentada y el desarrollo de nuevos procesos para la norma NMX-I-059/03-NYCE-2005 dentro 
del Centro de Desarrollo de Software de la Universidad Tecnológica del Centro de Veracruz.

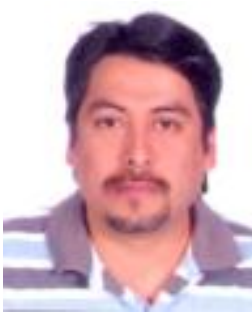
Juan Manuel Padilla Flores La Universidad Autónoma Metropolitana me otorgo el grado de Doctor en Ciencias Química en el año del 2010. Realice tres estancias posdoctorales en la universidad del Papaloapan, en la Universidad Autónoma Metropolitana y la Universidad de Guadalajara respectivamente, he impartido clases a nivel Licenciatura y posgrado desde 1995 a la fecha en la universidad Autónoma Metropolitana, Universidad del Papaloapan y actualmente en la Universidad Tecnológica Del Centro de Veracruz soy miembro activo del SNI nivel 1 cuento con 10 artículos publicados en revistas de impacto con alrededor de 70 citas y ocupo el puesto de jefe de investigadores.
\end{abstract}

\title{
(c) (i) (2)(2)
}

Esta obra está bajo una licencia de Creative Commons Reconocimiento-NoComercial-Compartirlgual 2.5 México. 